\title{
Transverse Beam Profile Measurement using Scraper Scans
}

\author{
Steven J. Werkema \\ September 13, 2001
}

A scraper scan - sending a scraper through a particle beam while measuring the intensity as a function of scraper position - is a common method of determining the profile of the beam. At first glance, this seems to be a rather simple procedure. Nevertheless, some care is required in the acquisition of the data and in the analysis if one is going to achieve an accurate result.

\section{Theoretical Overview}

Consider the transverse motion of a single beam particle at the longitudinal location of the scraper. The quantity to be measured - the transverse emittance of the beam - is the area of the phase space trajectory that contains $95 \%$ of the beam. An example of motion in $x-x^{\prime}$ coordinates is shown in Figure 1.

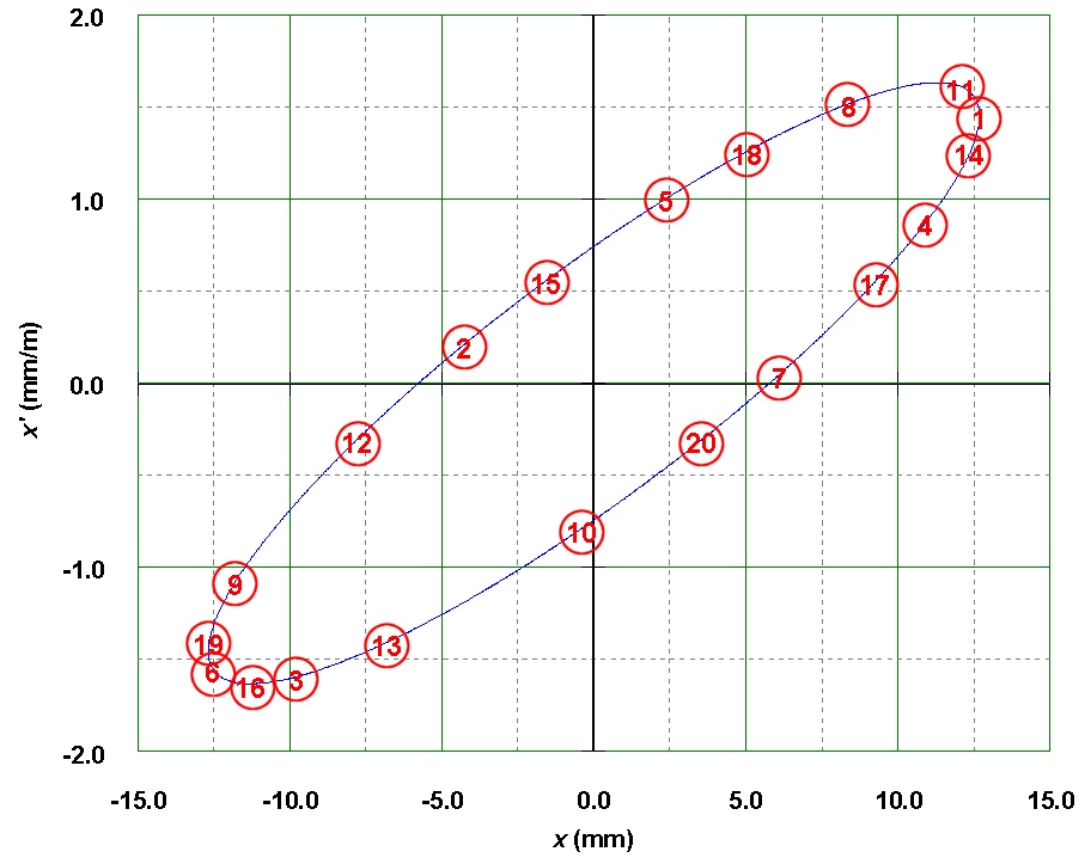

Figure 1 The motion of a particle of emittance $3.0 \pi \mathrm{mm}$-mrad in horizontal phase space at scraper A:RJ500 in the Accumulator. The red numbers indicate the turn number for 20 consecutive beam turns.

The basic procedure in what follows will be to transform the equations of motion to convenient coordinates, write down the two-dimensional transverse distribution of the beam, and relate that to the derivative of the beam intensity with respect to scraper position as the beam is scraped to extinction.

\section{A. Equations of transverse motion}

If betatron coupling can be neglected, the transverse motion is derived from the following Hamiltonian:

$$
H=\frac{1}{2} x^{\prime 2}+\frac{1}{2} K(s) x^{2}
$$


Here, the prime symbol (') denotes differentiation with respect to the longitudinal coordinate, $s$. $x^{\prime}$ is the dynamical variable that is canonically conjugate to $x$. Application of Hamilton's equations to equation (1) yields the familiar equation governing the linear transverse motion of the beam in an alternating gradient accelerator - Hill's equation: $x^{\prime \prime}+K x=0$.

Equation (2) gives a solution to Hill's equation that explicitly shows the variation of $x(s)$ with turn number $(n)$.

$$
x_{n}(s)=a \sqrt{\beta(s)} \cos \left(\mu(s+n L)-\mu_{0}\right)
$$

$L$ is the circumference of the ring. $\mu(s)$ is the betatron phase, given by:

$$
\begin{aligned}
\mu(s+n L) & =\mu_{0}+\int_{s_{0}}^{s+n L} \frac{d t}{\beta(t)} \\
& =\mu(s)+2 \pi n v
\end{aligned}
$$

$v$ is the betatron tune. The conjugate momentum, $x^{\prime}$, is found by taking the derivatives of equations (2) and (3) with respect to $s$, yielding:

$$
x_{n}^{\prime}=-\frac{a}{\sqrt{\beta}}\left[\alpha \cos \left(\mu(s+n L)-\mu_{0}\right)+\sin \left(\mu(s+n L)-\mu_{0}\right)\right]
$$

where $\alpha \equiv-\beta / 2$. The area of the ellipse traversed by a beam particle in $\left(x, x^{\prime}\right)$ phase space is a dynamical invariant given by ${ }^{1}$ :

$$
\text { Area }=\pi a^{2}=\gamma x^{2}+2 \alpha x x^{\prime}+\beta x^{\prime 2}
$$

where $\gamma=\frac{1+\alpha^{2}}{\beta}$.

\section{B. Transformation to $(\eta, \dot{\eta})$ Coordinates}

Since the area is an invariant, its value is preserved in canonical transformations. Hence, it is useful at this point to find a canonical transformation from $\left(x, x^{\prime}\right)$ coordinates to new $(\eta, \dot{\eta})$ coordinates such that the transformed Hamiltonian is of the following form ${ }^{2}$ :

$$
K=\frac{1}{2} \dot{\eta}^{2}+\frac{1}{2} \eta^{2}
$$

The phase space trajectory in $(\eta, \dot{\eta})$ coordinates is a circle. Since the phase space area is a dynamical invariant, the area of the circle in $(\eta, \dot{\eta})$ phase space is the same as the area of the ellipse in $\left(x, x^{\prime}\right)$ coordinates. The transformation that accomplishes this is ${ }^{3}$ :

$$
\begin{aligned}
& \eta=\frac{x}{\sqrt{\beta}} \\
& \phi=\mu(s)-\mu_{0}
\end{aligned}
$$

\footnotetext{
${ }^{1}$ See, for example, D.A. Edwards and M.J. Syphers, "An Introduction to the Physics of High Energy Accelerators" section 3.2.4.

${ }^{2} \dot{\eta}$ should not be confused with $\eta^{\prime}$. $\dot{\eta}$ is defined in equation (8). For the moment, one can view $\dot{\eta}$ merely as a symbol representing the canonical momentum without necessarily connecting it with a derivative.

${ }^{3}$ The generating function for this transformation is:

$$
\begin{array}{r}
F=F_{2}(x, \dot{\eta})-\eta \dot{\eta} \\
F_{2}(x, \dot{\eta})=-\frac{\alpha}{2 \beta} x^{2}+\frac{\dot{\eta} x}{\sqrt{\beta}}
\end{array}
$$


Combining equations (2) and (7) gives ${ }^{4}$ :

$$
\begin{aligned}
& \eta_{n}=a \cos \phi_{n} \\
& \dot{\eta}_{n} \equiv \frac{d \eta_{n}}{d \phi_{n}}=-a \sin \phi_{n}
\end{aligned}
$$

where $\phi_{n}=\mu(s)-\mu_{0}+2 \pi n v$. The trajectory of a particle in $(\eta, \dot{\eta})$ coordinates is a circle of radius $a$. The area of the phase space trajectory is $\pi a^{2}$, as expected. If $a_{95}$ denotes the phase space circle containing $95 \%$ of the beam, then the emittance, $\varepsilon$, is given by $\varepsilon=\pi a_{95}^{2}$.

\section{Determination of the Transverse Distribution of the Beam}

It is assumed in what follows that, prior to scraping, the transverse distribution of the beam is static at the location of the scraper. The transverse beam distribution can be written as $N(\eta, \dot{\eta})$, where $N(\eta, \dot{\eta}) d \eta d \dot{\eta}$ gives the number of beam particles in the phase space element between $(\eta, \dot{\eta})$ and $(\eta+d \eta, \dot{\eta}+d \dot{\eta})$. The $\eta$ distribution of the beam is given by:

$$
\frac{d N(\eta)}{d \eta}=\int_{-\infty}^{\infty} N(\eta, \dot{\eta}) d \dot{\eta}
$$

$d N(\eta)$ gives the number of particles with $\eta$ between $\eta$ and $\eta+d \eta$.

It is more convenient to deal with the amplitude, $a$, than $\dot{\eta}$. Using $\eta^{2}+\dot{\eta}^{2}=a^{2}$, the distribution function $N(\eta, a) d a$ is determined from $N(\eta, \dot{\eta}) d \dot{\eta}$ as follows:

where $N(\eta, a)=N(\eta, \dot{\eta}(\eta, a))$. Equation (9) becomes:

$$
\begin{aligned}
N(\eta, \dot{\eta}) d \dot{\eta} & =N(\eta, a) \frac{\partial(\eta, \dot{\eta})}{\partial(\eta, a)} d a \\
& =N(\eta, a) \frac{2 a}{\sqrt{a^{2}-\eta^{2}}} d a \quad(a \geq|\eta|)
\end{aligned}
$$

$$
\frac{d N(\eta)}{d \eta}=2 \int_{|\eta|}^{\infty} \frac{N(\eta, a)}{\sqrt{a^{2}-\eta^{2}}} a d a
$$

What remains is to relate $N(\eta, a)$ to what is measured in a scraper scan. The problem is greatly simplified by the observation that $N(\eta, a)$ depends only on the amplitude, $a$. This can be seen as follows: Let $n(\eta, a) \equiv N(\eta, a) / N_{0}$, where $N_{0}$ is the total number of beam particles. $n(\eta, a) d \eta d a$ is the joint probability of a beam particle with $\eta$ between $\eta$ and $\eta+d \eta$ and with $a$ between $a$ and $a+d a$. If $\mathcal{G}(a)$ is the probability distribution of $a$ and $\mathcal{F}(\eta \mid a)$ is the conditional probability distribution of $\eta$ for a given value of $a$, then

$$
n(\eta, a) d \eta d a=[\mathcal{F}(\eta \mid a) d \eta] \cdot[\mathcal{G}(a) d a]
$$

Since $\eta=a \cos \phi, \mathcal{F}(\eta \mid a)$ depends on $a$ and $\phi$. The $\phi$ values of the particles in the beam, due to filamentation, are uniformly distributed between 0 and $2 \pi$. Thus, $\eta$ is uniformly distributed between $-a$ and $a$. Therefore, $\mathcal{F}(\eta \mid a)$ is given by:

$$
\mathcal{F}(\eta \mid a)=\left\{\begin{array}{cc}
\frac{1}{2 a} & -a \leq \eta \leq a \\
0 & |\eta|>a
\end{array}\right.
$$

\footnotetext{
${ }^{4}$ Note: From equation (8): $\ddot{\eta}=-a \cos \phi=-\eta$. This also follows from the application of Hamilton's equations to equation (6).
} 
$n(\eta, a)$ (and hence $N(\eta, a)$ ) depends only on $a$. Thus,

Therefore,

$$
n(\eta, a)=\mathcal{F}(\eta \mid a) \cdot \mathcal{G}(a) \equiv\left\{\begin{array}{cc}
\frac{g(a)}{N_{0}} & |\eta| \leq a \\
0 & |\eta|>a
\end{array}\right.
$$

$$
N(\eta, a)=\left\{\begin{array}{cc}
g(a) & |\eta| \leq a \\
0 & |\eta|>a
\end{array}\right.
$$

$g(a)$ is the amplitude distribution function. The number of particles, $d N(a)$, with amplitudes between a and $a+d a$ is given by: $d N(a)=g(a) \bullet 2 \pi a d a$ (see Figure 2).

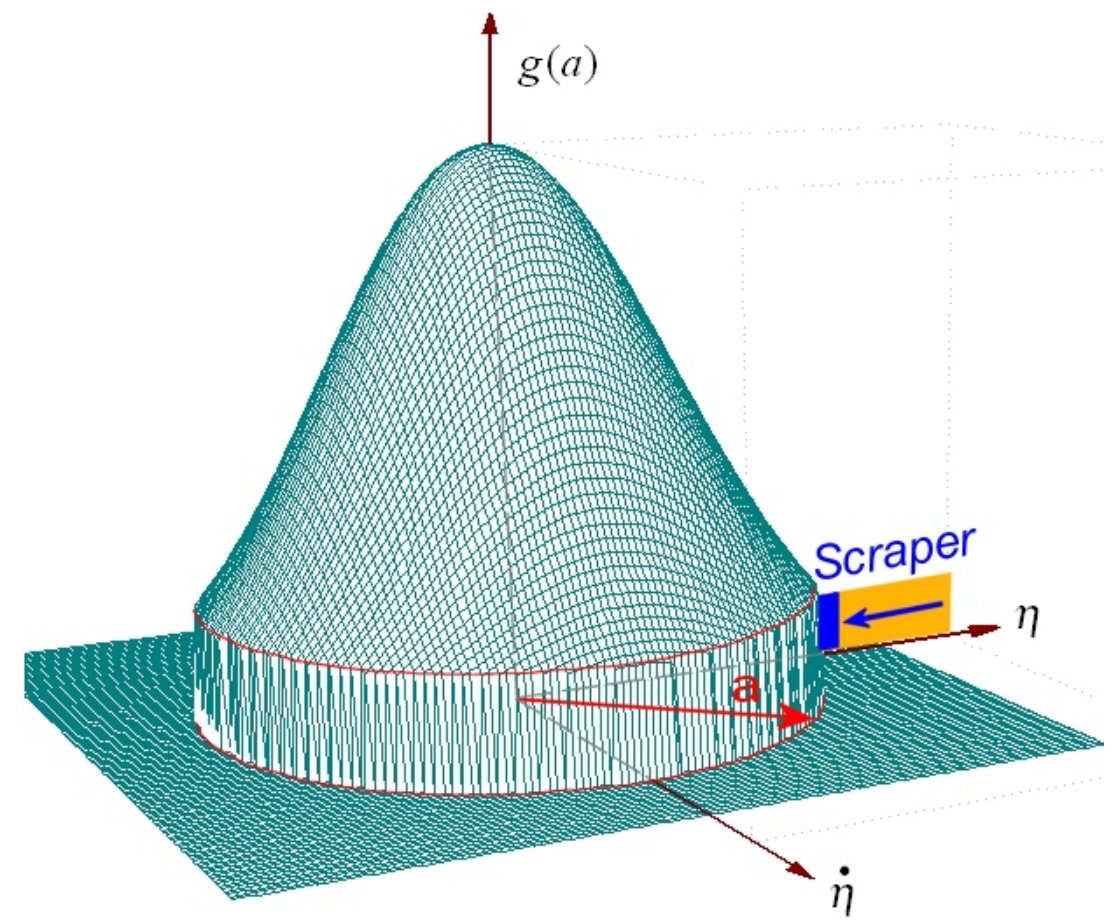

Figure 2 The two-dimensional distribution of the beam in $(\eta, \dot{\eta})$ coordinates as the beam is scraped. The time that the scraper remains in the neighborhood of each amplitude is long enough for all the particles oscillating with that amplitude to interact with the scraper and be lost from the beam.

If $x_{\mathrm{s}}$ is the instantaneous position of the scraper, then $a_{s}=\beta^{-1 / 2} x_{s}$ is the corresponding scraper amplitude. The quantity that is measured during a scraper scan is the amount beam remaining as a function of $x_{\mathrm{s}}, I\left(a_{S}\right)=I\left(\beta^{-1 / 2} x_{S}\right) . I\left(a_{s}\right)$ is given by:

$$
I\left(a_{s}\right)=2 \pi \int_{0}^{a_{S}} g(a) a d a
$$

Figure 3 shows a plot of $I\left(a_{s}\right)$ from data taken during an actual scraper scan.

The amplitude distribution is obtained by differentiation of equation (16).

$$
g(a)=\frac{1}{2 \pi a}\left[\frac{d I(a)}{d a}\right]
$$

The quantity in brackets in equation (17) is obtained by differentiation if the $I\left(a_{s}\right)$ data obtained during the scraper scan. Figure 4 shows a plot of the $g(a)$ distribution function that is derived by differentiation of the $I\left(a_{s}\right)$ data in Figure 3. 
Pbar Note 665

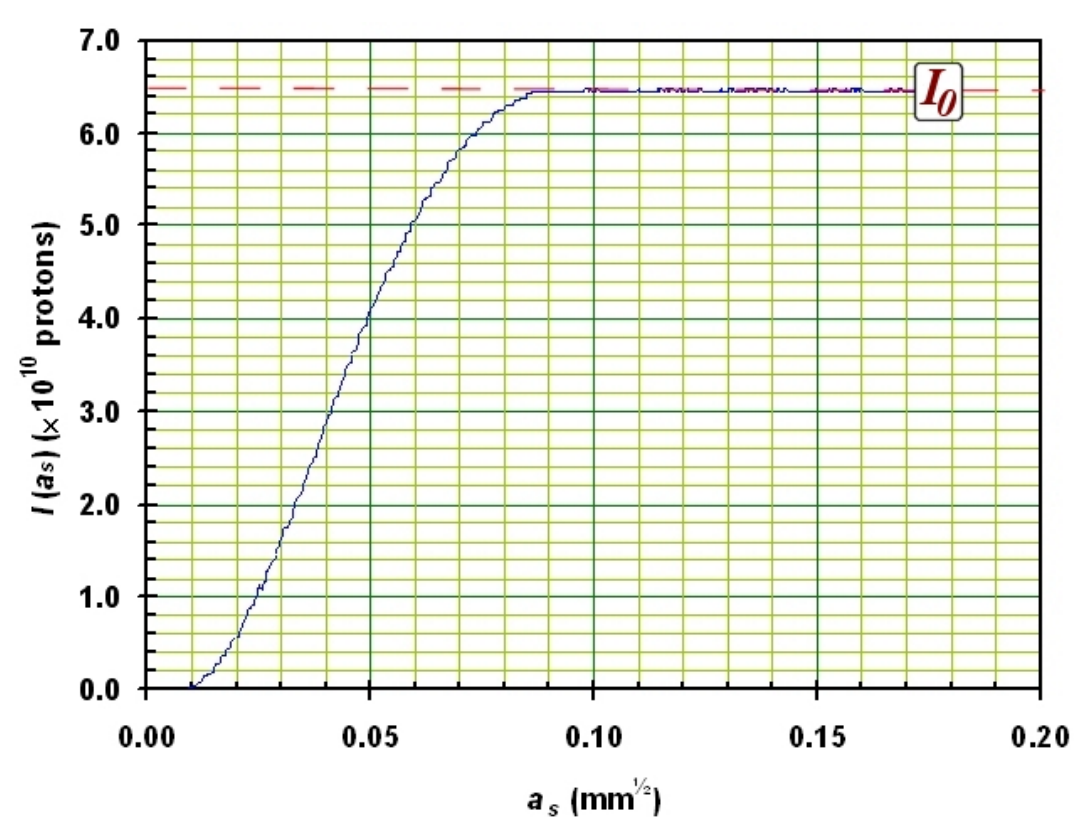

Figure $3 I\left(a_{s}\right)$ versus $a_{s}$ for a vertical scraper scan of proton beam in the Accumulator.

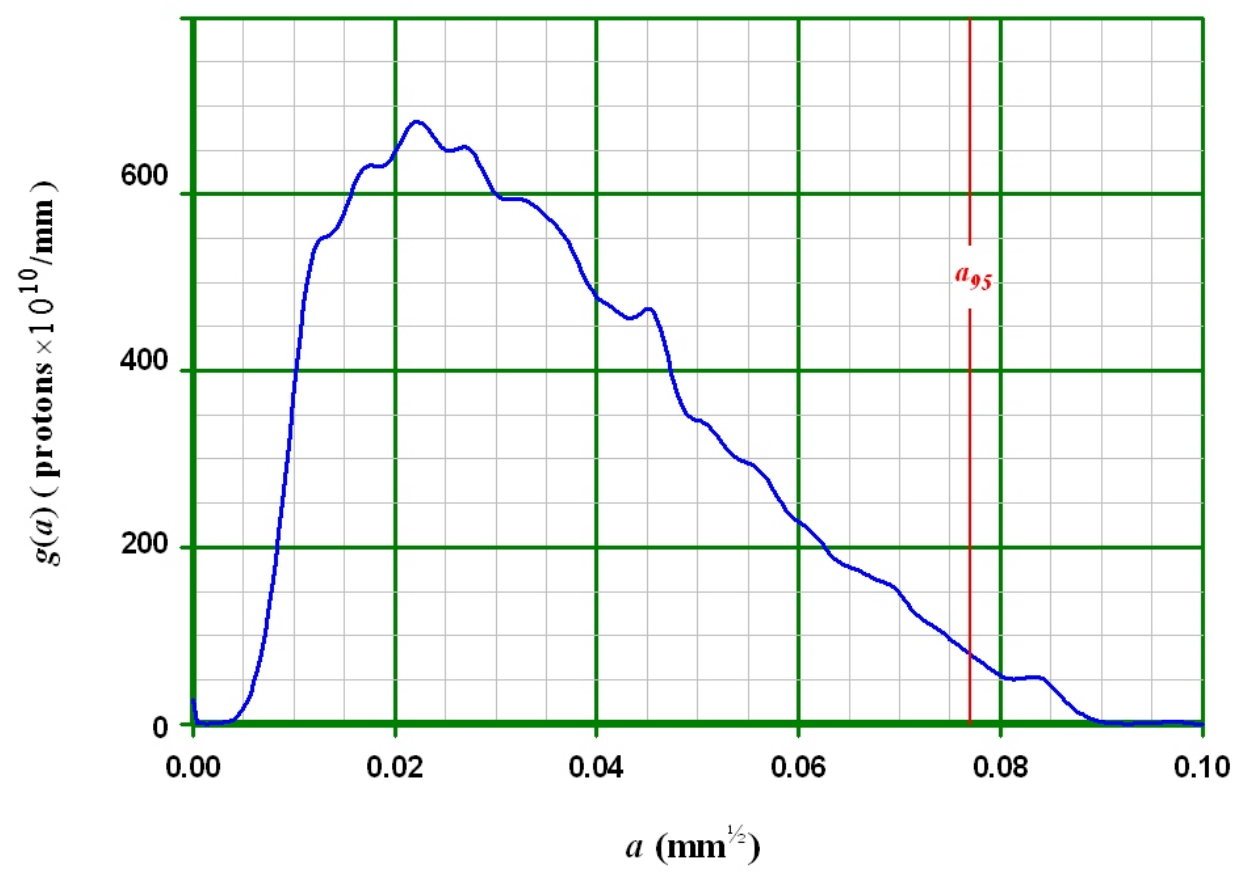

Figure 4 The amplitude distribution function, $g(a)$, derived from an analysis of the scraper scan shown in Figure 3. The "hole" in the distribution at small amplitudes seems to be a consequence of heating the beam prior to the scraper scan.

If $I_{0}$ is the beam intensity prior to the scraper scan, the emittance of the beam is determined by solving for $a_{95}$ - the value of $a$ for which $I(a)=0.95 \bullet I_{0} . a_{95}$ is given by:

$$
0.95 I_{0}=2 \pi \int_{0}^{a_{95}} g(a) a d a
$$


Once $a_{95}$ is determined, the emittance, $\varepsilon$, is then given by:

$$
\varepsilon=\pi a_{95}^{2}
$$

Once $g(a)$ is known, the $\eta$ distribution of the beam can be calculated by substitution of equation (15) into (11). The $x$ distribution can then be calculated from the $\eta$ distribution by:

$$
\begin{aligned}
\frac{d N(x)}{d x} & =\frac{d \eta}{d x} \frac{d N(\eta(x))}{d \eta} \\
& =\frac{1}{\sqrt{\beta}} \frac{d N\left(x \beta^{-1 / 2}\right)}{d \eta}
\end{aligned}
$$

The resulting $x$ distribution is given by:

$$
\frac{d N(x)}{d x}=2 \int_{\left|x \beta^{-1 / 2}\right|}^{\infty} \frac{g(a)}{\sqrt{\beta a^{2}-x^{2}}} a d a
$$

Figure 5 shows the $\mathrm{x}$ distribution derived from the amplitude distribution shown in Figure 4.

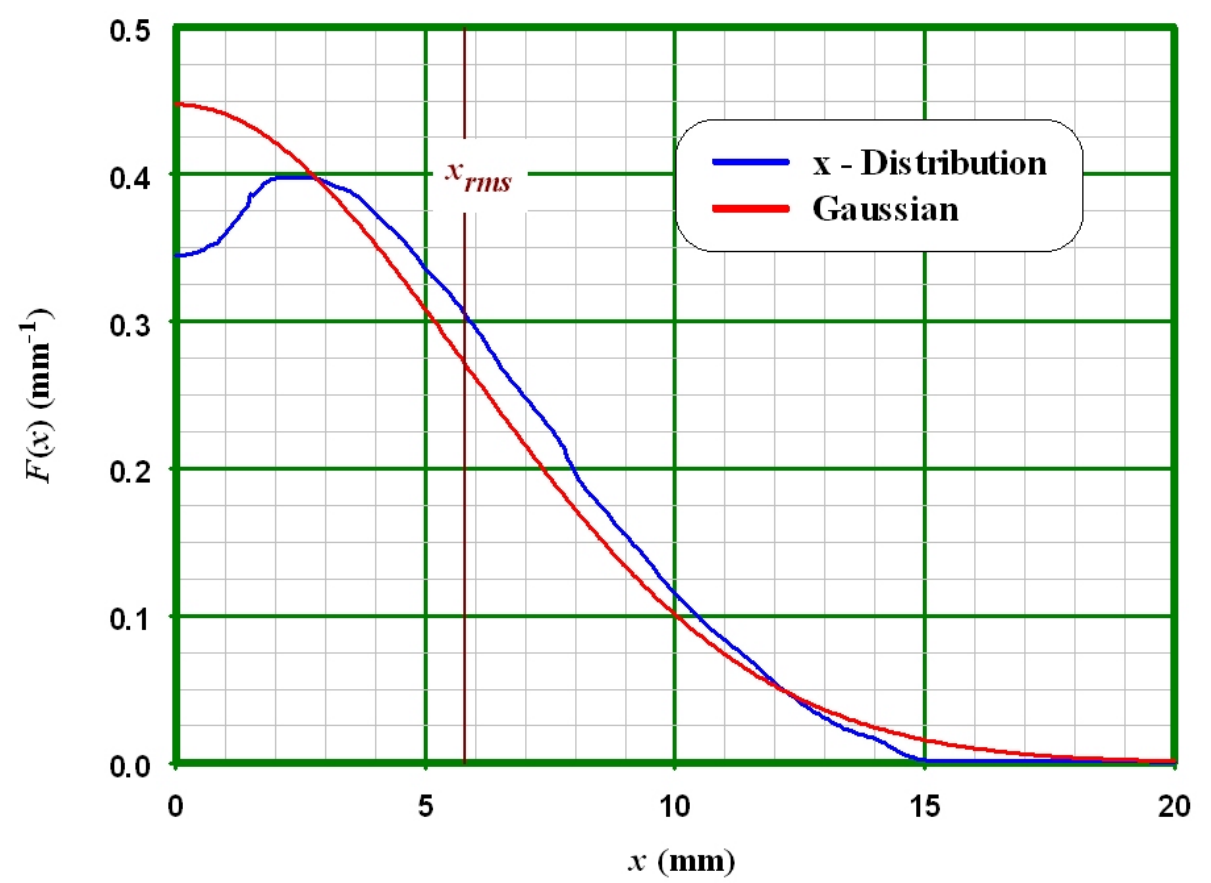

Figure $5 x$-distribution of the beam calculated from the $g(a)$ given in Figure 4 . The red line is a gaussian with $\sigma$ equal to the root-mean-square of the $x$-distribution. 


\section{Analysis of scraper scans performed in August and September of 2001}

The analysis of scraper scan data taken during two beam study periods on August 29, 2001 and September 9,2001 is presented here. Table 1 shows the relevant beam conditions pertinent to each scan. The beam current readouts differ in precision and update rate. A:IBEAM is a high precision beam current readout but its update rate was too slow to keep up with the scraper movement. The update rate of A:IBEAMV is adequate, however it is somewhat lacking in precision. The results of the analysis of these scans are summarized below in Table 2.

Table 1: Scraper Scan Conditions

\begin{tabular}{|l|c|c|}
\hline & August 29 Scans & September 9 Scans \\
\hline Orbit & Extraction Orbit & Core Orbit \\
\hline Beam heated prior to scan & No & Yes \\
\hline Beam Current Readout & A:IBEAM & A:IBEAMV \\
\hline
\end{tabular}

The amplitude and $x$ distributions are given in Figure 6 through Figure 9 below.
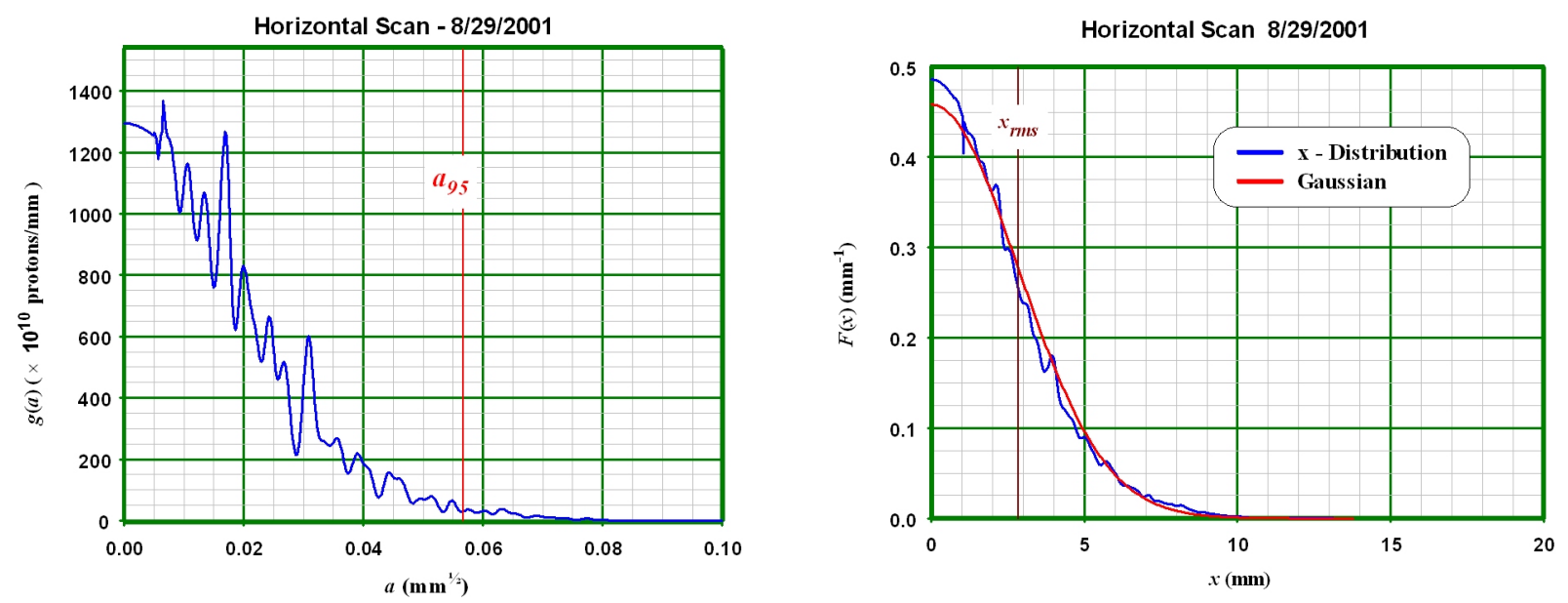

Figure 6 The amplitude $(a)$ distribution and the $x$ distribution, $F(x)$, for the horizontal scraper scan of August 29, 2001. The red trace on the $F(x)$ graph is a gaussian with $\sigma=x_{r m s}$.
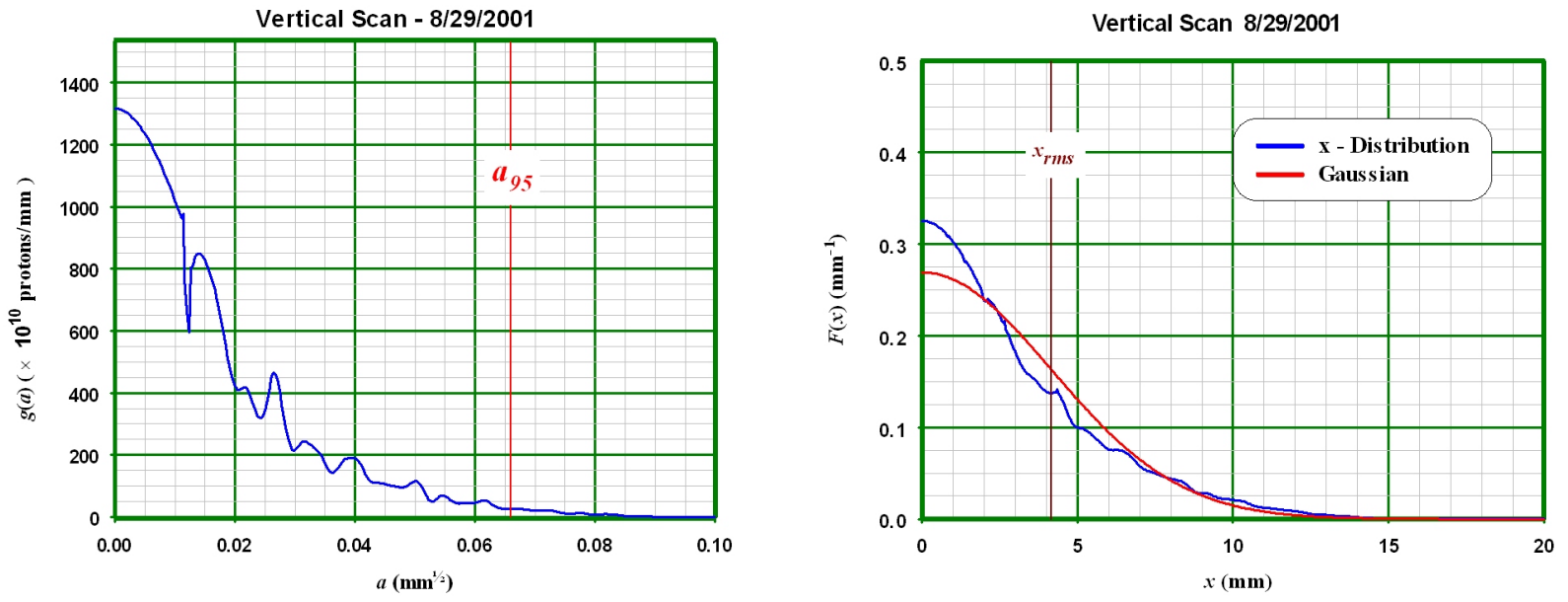

Figure 7 The amplitude (a) distribution and the $x$ distribution, $F(x)$, for the vertical scraper scan of August 29, 2001. The red trace on the $F(x)$ graph is a gaussian with $\sigma=x_{r m s}$. 

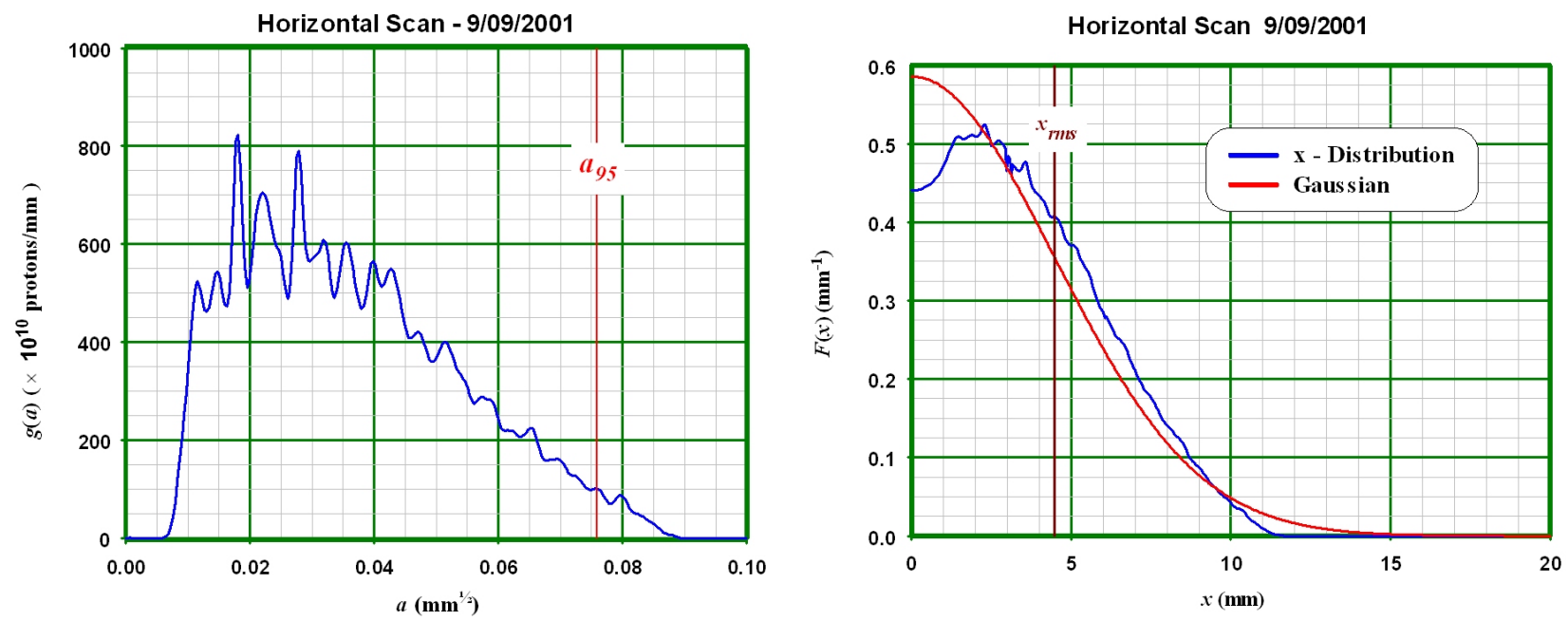

Figure 8 The amplitude (a) distribution and the $x$ distribution, $F(x)$, for the horizontal scraper scan of September 9, 2001. The red trace on the $F(x)$ graph is a gaussian with $\sigma=x_{r m s}$. The "hole" in the distribution for $a<0.01 \mathrm{~mm}{ }^{1 / 2}$ is probably caused by heating the beam prior to the scraper scan.
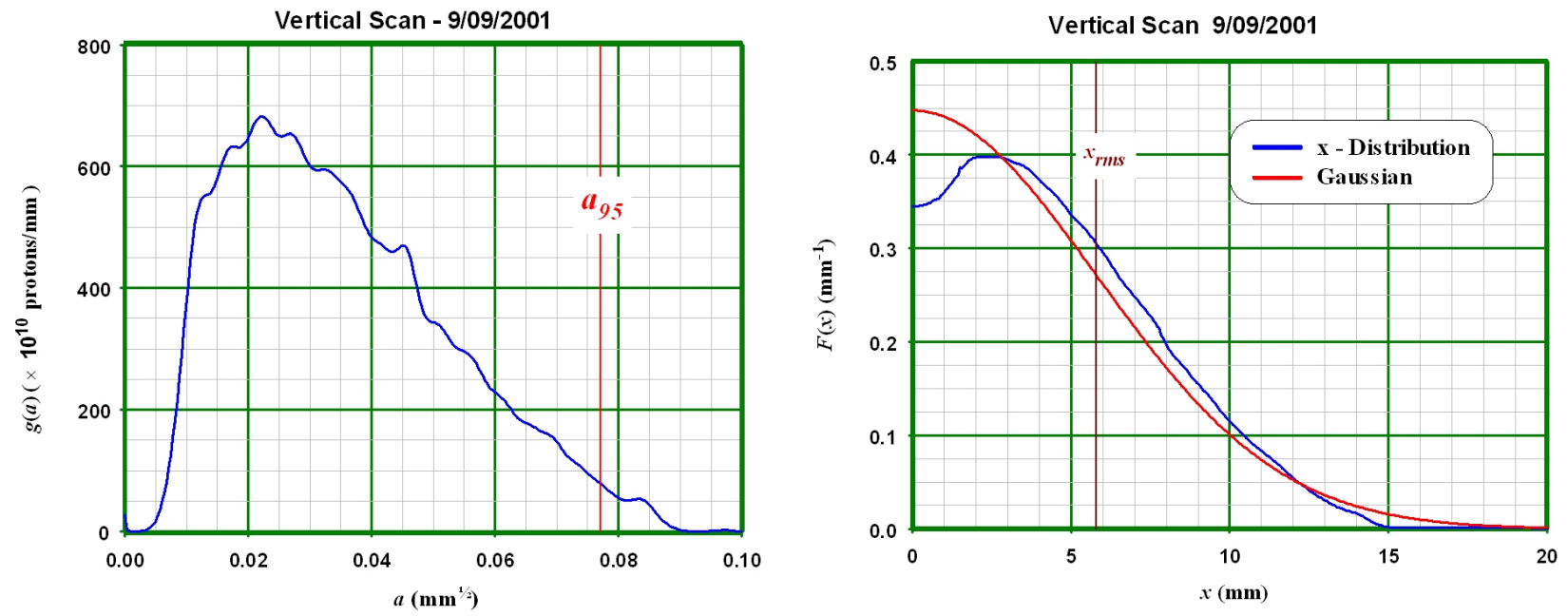

Figure 9 The amplitude $(a)$ distribution and the $x$ distribution, $F(x)$, for the vertical scraper scan of September 9, 2001. The red trace on the $F(x)$ graph is a gaussian with $\sigma=x_{r m s}$. The "hole" in the distribution for $a<0.01 \mathrm{~mm}{ }^{1 / 2}$ is probably caused by heating the beam prior to the scraper scan.

Table 2: Scraper Scan data and Results

\begin{tabular}{|c|c|c|c|c|c|c|}
\hline Date & Plane & $\begin{array}{c}\text { A:IBEAM } \\
(\mathbf{m A})\end{array}$ & $\begin{array}{c}\text { A:EMITH(V) } \\
\pi \text { mm-mrad-mA }\end{array}$ & $\begin{array}{c}\text { A:EMITH(V)N } \\
\pi \text { mm-mrad }\end{array}$ & $\begin{array}{c}\boldsymbol{f}_{\text {rev }} \\
\mathbf{( H z )}\end{array}$ & $\begin{array}{c}\varepsilon=\pi \boldsymbol{a}_{95}^{2} \\
\pi \text { mm-mrad }\end{array}$ \\
\hline $\mathbf{8 / 2 9 / 2 0 0 1}$ & $\mathrm{H}$ & 3.278 & 8.352 & 2.547 & 628764.7 & 3.213 \\
\hline $\mathbf{8 / 2 9 / 2 0 0 1}$ & $\mathrm{V}$ & 2.808 & 7.488 & 2.667 & 628764.8 & 4.352 \\
\hline $\mathbf{9 / 0 9 / 2 0 0 1}$ & $\mathrm{H}$ & 6.656 & 23.29 & 3.552 & 628887.7 & 5.751 \\
\hline $\mathbf{9 / 0 9 / 2 0 0 1}$ & $\mathrm{V}$ & 6.529 & 27.44 & 4.202 & 628887.5 & 5.935 \\
\hline
\end{tabular}

The most obvious use of the information in Table 2 is for the calibration of the Accumulator emittance monitors. The use of scraper scans to calibrate the emittance monitors must be approached carefully. The inputs to the Accumulator emittance monitors are transverse schottky pickups that are resonant at $79 \mathrm{MHz}$. The schottky signal from one of these pickups is 
proportional to the root-mean-square dipole moment of the beam. Thus, the output of the emittance monitors can be written as:

$$
V_{\varepsilon} \propto\left\langle d^{2}\right\rangle=\left\langle x^{2}\right\rangle I_{b}
$$

where $d$ is the dipole moment of the beam and $I_{b}$ is the beam current. The $95 \%$ emittance can be written as:

$$
\begin{aligned}
\varepsilon_{95} & =\frac{2 \pi \ln (0.05)}{\beta_{s c}} F\left\langle x^{2}\right\rangle \\
& =F \varepsilon_{g}
\end{aligned}
$$

$\beta_{s c}$ is the beta-function at the schottky detector and $F$ is a form factor that is equal to one if the transverse distribution is a gaussian. $\varepsilon_{g}$ is the $95 \%$ emittance beam with a gaussian transverse distribution.

Calibration of the emittance monitors involves writing the measured emittance in terms of the output of the emittance monitor. If $k$ is the proportionality constant between the emittance monitor output and the rms dipole moment of the beam, then the $95 \%$ emittance can be written as:

$$
\begin{aligned}
\varepsilon_{95} & =\frac{2 \pi \ln (0.05)}{\beta_{s c}} F \frac{V_{\varepsilon}}{k I_{b}} \\
& =K F \frac{V_{\varepsilon}}{I_{b}}
\end{aligned}
$$

$K$ is a constant depending only on numerical factors and the beta-function at the schottky pickup. Since the emittance monitor is sensitive to only one moment of the beam distribution, some assumptions about the shape of the transverse distribution must be made (i.e. one must pick a value for $F$ ). Generally, a gaussian distribution is assumed, thus setting $F=1$. Table 3 shows that this assumption gives rise to an uncertainty in the measurement of the emittance that can be as large as $20 \%$.

Table 3: Form factors from Scraper Scan data

\begin{tabular}{|c|c|c|c|c|}
\hline Date & Plane & $\begin{array}{c}\varepsilon=\pi \boldsymbol{a}_{95}^{2} \\
\pi \text { mm-mrad }\end{array}$ & $\begin{array}{c}\boldsymbol{\varepsilon}_{\boldsymbol{g}} \\
\pi \text { mm-mrad }\end{array}$ & $\boldsymbol{F}$ \\
\hline $\mathbf{8 / 2 9 / 2 0 0 1}$ & $\mathrm{H}$ & 3.213 & 2.811 & 1.143 \\
\hline $\mathbf{8 / 2 9 / 2 0 0 1}$ & $\mathrm{V}$ & 4.352 & 3.626 & 1.200 \\
\hline $\mathbf{9 / 0 9 / 2 0 0 1}$ & $\mathrm{H}$ & 5.751 & 7.039 & 0.817 \\
\hline $\mathbf{9 / 0 9 / 2 0 0 1}$ & $\mathrm{V}$ & 5.935 & 7.036 & 0.843 \\
\hline
\end{tabular}

